\title{
Clinical Pharmacist Integration Into Veterans' Primary Care: Team Members Perspectives
}

\author{
Anna Zogas, PhD, Chris Gillespie, PhD, Felicia Kleinberg, MSW, Joel I. Reisman, AB, \\ Ndindam Ndiwane, MPH, Michael H. Tran, PharmD, BCPS, \\ Heather L. Ourth, PharmD, BCPS, BCGP, FASHP, \\ Anthony P. Morreale, PharmD, MBA, BCPS, FASHP, Donald R. Miller, ScD, and \\ Megan B. McCullough, PhD
}

Background: With the restructuring of primary care into patient-centered medical homes (PCMH), researchers have described role transformations that accompany the formation of core primary care teamlets (eg, primary care provider, registered nurse care manager, licensed practical nurse, medical support assistant). However, few studies offer insight into how primary care teamlets, once established, integrate additional extended team members, and the factors that influence the quality of their integration.

Methods: We examine the process of integrating Clinical Pharmacy Specialists (CPS) into primary care teams in the Veterans Health Administration (VHA). We conducted semi-structured interviews with $\mathrm{CPS}(\mathrm{n}=$ 6) and clinical team members $(n=16)$ and performed a thematic analysis of interview transcripts.

Results: We characterize 2 ways CPS are integrated into primary care teamlets: in consultative roles and collaborative roles. CPS may be limited to consultative roles by team members' misconceptions about their competencies (ie, if CPS are perceived to handle only medication-related issues like refills) and by primary care providers' opinions about distributing responsibilities for patient care. Over time, teams may correct misconceptions and integrate the CPS in a more collaborative role (ie, CPS helps manage disease states with comprehensive medication management).

Conclusions: CPS integrated into collaborative roles may have more opportunities to optimize their contributions to primary care, underscoring the importance of clarifying roles as part of adequately integrating advanced practitioners in interprofessional teams. (J Am Board Fam Med 2021;34:320-327.)

Keywords: Clinical Pharmacists, Disease Management, Interprofessional Relations, Medical Home, Medication Therapy Management, Military Medicine, Patient Care Team, Pharmacists, Primary Care Physicians, Primary Health Care, Qualitative Research, Veterans Health

\section{Introduction}

Primary care is undergoing a widespread reorganization into patient-centered medical homes $(\mathrm{PCMH})$,

This article was externally peer reviewed.

From the Center for Healthcare Organization and Implementation Research, VA Boston Healthcare System, MA (AZ); Center for Healthcare Organization and Implementation Research, VA Bedford Healthcare System, MA (CG, FK, JIR, NN, DRM, MBM); Pharmacy Benefits Management Services, National Clinical Pharmacy Practice Office, US Department of Veterans Affairs, Washington, DC (MHT, HLO, APM); University of Massachusetts, Lowell, Center for Population Health (DRM); University of Massachusetts, Lowell, Zuckerberg School of Health Sciences, Department of Public Health (MBM).

Funding: This study was funded by the US Department of Veterans Affairs Office of Rural Health and the Clinical Pharmacy Practice Office of the Pharmacy Benefits Management Service (PI: MBM). The work and the writing of this manuscript were supported by the Department of where a primary care provider leads a team who collectively take responsibility for patients' care. ${ }^{1}$ In the Veterans Health Administration (VHA) PCMH are called Patient Aligned Care Teams (PACT), with core teamlets comprised of a primary care provider (PCP) (physician, physician assistant, or a nurse practitioner), a registered nurse ( $\mathrm{RN})$ care manager, a

Veterans Affairs Office of Academic Affiliations Advanced Fellowship Program in Health Services Research, and the Center for Healthcare Organization and Implementation Research (CHOIR), VA Boston Healthcare System, Boston, MA (AZ).

Conflict of interest: None.

Corresponding author: Megan B. McCullough, PhD, Center for Healthcare Organization and Implementation Research, VA Bedford Healthcare System, (152) 200 Springs Road, Bedford, MA 01730, USA (781) 687-4503. (E-mail: Megan.McCullough@va.gov). 
licensed practical nurse (LPN), and a clerk or medical support assistant. The core teamlets are grouped into larger teams, which include 1 or more extended team members, such as clinical pharmacists, psychologists, social workers, health coaches, and dieticians. ${ }^{2}$ In this arrangement, interprofessional teamwork is central to the delivery of care. ${ }^{3-5}$

When providers trained in different professions come together as a team, they face a need to define or clarify how each person will participate in daily clinic operations. ${ }^{6,7}$ With well-defined roles, each member understands their own and others' responsibilities, which can ensure efficient patient management and reduce confusion and power struggles. ${ }^{6,8}$ Roles can be codified in professional licenses and scopes of practice, but even roles defined at the structural level are also negotiated at the interpersonal level. ${ }^{9,10}$ Researchers studying Canadian primary care teams, for instance, found role clarification to be influenced by team dynamics (eg, leadership within the team) and individual dynamics (eg, personality; competence at negotiation). ${ }^{8,9}$ Likewise, during the early implementation of PACT in the VHA (during 2010 to 2011) teamlevel negotiations over roles were essential to how teams negotiated sharing work. In some teamlets, members actively shaped their roles through mutual teaching, learning, accepting work, delegating, taking initiative, and negotiating cross-disciplinary role agreement, which helped optimize members' skills. ${ }^{11,12}$ At the same time, teamlets in which PCPs struggled to delegate and share tasks with $\mathrm{RNs}$ and clinical staff experienced a lack of cohesion and struggles to optimize members' skills. ${ }^{13-17}$

In the VHA, established PACT teams are in the process of negotiating roles for a new member: pharmacists with advanced training, called Clinical Pharmacy Specialists (CPS). ${ }^{18}$ CPS are advanced practice providers with the authority to prescribe and modify medications, but they do not diagnose, so PCP-pharmacist collaboration is essential. ${ }^{18,19}$ CPS have worked in the VHA since the early 1990s, primarily managing diseases (eg, diabetes, hypertension, and lipids) in isolation, or specific medications (eg, warfarin). ${ }^{20-22}$ However, CPS' training also prepares them to manage patients' multiple disease states using comprehensive medication management (CMM), which includes assessing patients' medication needs, adding drug therapies as necessary, titrating doses to achieve therapeutic levels, and assessing the appro- priateness, effectiveness, and safety across all their medications (prescription, nonprescription, alternative, traditional, vitamins, and nutritional supplements). ${ }^{23}$ CPS' provision of these services has been shown to be a cost-effective ${ }^{24}$ way to improve population health and intermediate clinical outcomes in diabetes, ${ }^{25-27}$ hypertension, ${ }^{28}$ and hyperlipidemia. ${ }^{29}$ Evidence from 1 VA medical center demonstrated that approximately $27 \%$ of follow-up visits for patients with chronic illnesses in primary care could be shifted to the CPS, ${ }^{30}$ which may allow PCP effort to be directed toward other patient care goals.

In 2017, the VHA launched an initiative (the Clinical Pharmacy Specialist Rural Veteran Access Initiative [CRVA]) to improve patient access to primary care by introducing CPS into PACT to perform CMM and medication-related patient visits. $^{20,31}$ The initiative focused on diversifying CPS' roles beyond disease- and medication-specific medication management to CMM and providing comprehensive (rather than isolated) medication management. ${ }^{22}$ In this study, we analyze the complex process of integrating CPS into PACT. We analyze CPS' and clinical team members' perceptions of the CPS' role to better understand how the challenges and misconceptions that arise during the process of defining team members' roles influence CPS' ability to optimize CMM in primary care.

\section{Methods}

This study is part of a larger mixed-methods evaluation 2017 to 2020 of the VHA's CRVA. CPS $(\mathrm{n}=124)$ and their team members $(\mathrm{n}=1177)$ responded to an online survey that measured CPS' integration (using an instrument adapted from Farrell et $\mathrm{al}^{32}$ ). The design and findings of the larger evaluation have been reported as conference proceedings. ${ }^{33-35}$ Here, we focus exclusively on qualitative findings from primary care PACT. We purposively selected our interview sample based on organizational and individual factors, including the effectiveness of different medical center's implementation of the initiative, CPS' performance as measured by clinical pharmacy operational partners, team members' roles (eg, PCPs, RNs), and our assessment of the sample size necessary to reach theoretical saturation. ${ }^{36}$ Semi-structured, in-depth interviews were conducted via telephone after the CPS had been in their positions for at least 
6 months. Interviews explored the role of CPS from the perspectives of the CPS and the members of their interprofessional clinical teams.

The qualitative team included $3 \mathrm{PhD}$ level qualitative researchers (AZ, CG, MBM). Interviews were recorded and transcribed verbatim to facilitate qualitative data analysis. The team examined a subset of transcripts and used a process of critical review and consensus-building to develop and define codes and coding rules. Inductive codes, developed through thematic analysis, captured concepts related to integration and organizational attributes. ${ }^{37-40}$ We ensured analytic rigor through regular team meetings (for coding consistency), and the use of NVivo computerized software (to manage data analysis and facilitate comparisons between team members' coding and conclusions).

The evaluation of the CRVA initiative was reviewed by the Institutional Review Board at VA Bedford Healthcare System and determined to be quality improvement, exempting it from further oversight. Verbal assent was obtained from all participants.

\section{Results}

We interviewed CPS $(n=6)$ and clinical team members $(\mathrm{n}=16)$ (Table 1) between December 2017 and December 2018.

PACT members welcomed the addition of CPS as extended PACT team members. CPS delivered patient care (face-to-face, phone, video) and communicated regularly with team members (in person, by phone, instant message, and via the electronic medical record). When CPS joined primary care

Table 1. Clinical Staff of Veterans Health Affairs Medical Centers or Community-Based Outpatient Clinics, Interviewed December 2017 to December 2018

\begin{tabular}{lc}
\hline Role & No. of Participants \\
\hline CPS & 6 \\
PCPs (physician, PA, nurse practitioner) & 12 \\
Primary Care & $\mathbf{1 1}$ \\
Primary Care and Mental Health & $\mathbf{1}$ \\
Other team members (RN, LPN) & 4 \\
Total Interviews & 22 \\
\hline
\end{tabular}

CPS, clinical pharmacy specialist; PCPs, primary care physicians; PA, physician assistant; RN, registered nurse; LPN, licensed practical nurse. teams their role was ambiguous, the process of integration was nuanced and complicated, and there were misconceptions about clinically trained pharmacists. We demonstrate the nuances of integration by showing 2 ways that teams incorporated the CPS: consultative integration and collaborative integration.

\section{CPS Integrated in Consultative Roles}

In some teams, CPS' role was primarily supporting PCPs using their expertise in medications, drug interactions, and the VHA's prescription drug formulary.

It's absolutely a safety issue to have clinical pharmacists available to primary care doctors if we have questions about medications, interactions, changes, and alternatives. [...] You've got somebody who has a first-hand working knowledge of these medications as an expert. Team Member 19 (PCP)

Some PCPs viewed CPS as a resource for operational medication-related issues (eg, refills, renewals, tracking narcotics or mail-order) and for answering targeted medication questions (eg, questions about pharmaco-dynamics, titration, or the results of toxicology tests):

I'm probably bothering [the CPS] like once every couple of weeks. Usually, it's about medication concerns-tapering or cross-tapering drugs. [...] I've tagged the CPS in several notes like that, about what can I do differently as far as medications. [...] [The CPS] is really good at getting back in touch with me and going over notes and giving recommendations about what I should maybe consider the next time I see a patient. Team Member 22 (PCP)

The same PCP expressed ambivalence about CPS' increased responsibility in primary care:

I have mixed feelings about that just because it's one more person in the mix and I would rather keep patients under my care versus sharing them with somebody else, just because it prevents too many cooks in the kitchen. I see the reasoning for it. I don't know if I completely am behind it. I think it can lead to fractured care if it's not being followed closely enough. Team Member 22 (PCP)

This view of PCP patient ownership positions the CPS as auxiliary support for the PCP. We characterize this as consultative integration.

CPS in consultative roles perceived providers as having misconceptions about CPS' capabilities, experience, and scope of practice. 
We still do get some of the generalized questions about medications from the pharmacy, you knowrefills. We are able help with that. However, sometimes they [team members] do miss that we are more than that - that we are more than just medication reconciliation or polypharmacy, we are actually providers that can prescribe and can adjust medication. CPS 2

This CPS contrasted their clinical role ("we are actually providers") with a well-known role of pharmacists, medication distribution ("just medication reconciliation or polypharmacy"). CPS noted that consultative roles limited their ability to contribute to the team's work when it manifests as a lack of referrals to the CPS for CMM. For instance, 1 CPS noted that PCPs' views of patient ownership may prevent them from delegating CMM duties to CPS:

One of my providers works with me the least. And that's mostly because he's been pretty outgoing with other providers about his disagreement with pharmacists managing patients. $\mathrm{He}$ just doesn't agree that the pharmacists have, I guess, the clinical knowledge, to change patients' medications. [...] I've worked with his team [for eight months], and he has literally never sent me a single patient. CPS 5

When CPS are integrated in this consultative role, PCPs may only infrequently refer patients to CPS for CMM, limiting the CPS' ability to help improve patients' access to care. In teams where the CPS was initially integrated primarily as a consultant, the role had not changed at the time we conducted interviews.

\section{Moving toward a Collaborative Role}

Some teams integrated CPS in roles that better facilitated their ability to practice CMM by starting, changing, or stopping medications, and therefore optimized their work as advanced practice providers. When teams incorporated CPS in this more expansive role, we characterize it as collaborative integration. Arriving at collaborative integration required moving through a period of role ambiguity and negotiating a more collaborative role for the CPS. One CPS described how their autonomy was negotiated over time:

At first, I felt like the folks [that] were the most receptive [were] the midlevel providers. Like the two PAs that I have. [...] Because maybe their pharmacotherapy background wasn't as strong, so they were really happy to have any help. And I think, still most of my referrals-maybe like 60/ 40 split-come from the mid-level providers versus the physicians. And at first, some of the physicians said, 'That's fine. You can change things. But cosign me to your notes, so I know what you did that day.' [...] Now it's completely deferred to 'Oh, let's just let the pharmacist handle that.' Complete confidence in me just addressing the issue. CPS 1

Negotiating and clarifying resulted in a more expansive role for the CPS, in which they became collaborators with PCPs. For example, 1 PCP described how the CPS' mission of CMM meaningfully contributed to improving patient access to care:

I have found them [CPS] to be an asset when it comes to disease management. Especially... with diabetes... They [CPS] have time, and they're able to give medication, which is very vital to management...and explaining to the patient about the disease process, about the medication, what to expect. ..CPS are another source of medical care. . .if patients [are] not able to reach their doctor...I think it gives better patient care to have a clinical pharmacist on board the teamlet. Team Member 16 (PCP)

Here we see that in an integrated team, a PCP sends appropriate patients to the CPS to get them focused care. Working with the CPS, the patient reaches the treatment goal, their disease state stabilizes, and they are sent back to the PCP. This collaborative dynamic then opens slots for the CPS to take on new patients struggling and in need of CMM.

Another clinical team member described the collaboration between team members as producing holistic care for the patient:

If we feel like someone's medications are not managing diabetes, that we know that she's [the CPS is] going to get in touch with that patient and keep up with it and help. [...] I feel that it is kind of a more of a holistic care where it is not just left up to 1 person. Team member 7 (LPN)

From CPS' perspective, being integrated into a collaborative role was professionally fulfilling because it allowed them to provide direct patient care managing a variety of disease states while feeling like members of the team. In response to being asked what brings professional satisfaction, 1 CPS replied:

It's definitely the patient interactions. And, being a part of the team with the doctors. Because I think in the past when I was in anticoag [the CPS-run Anticoagulation Clinic] it felt 
somewhat separated. As a PACT pharmacist, there is a lot more variety. CPS 6

When teamlets integrated the CPS as a full collaborator, CPS maximized their contributions to primary care by working to the top of their scope of practice and fully participating in managing disease states with CMM.

\section{Discussion}

Results indicate that CPS integration has been successful on many levels; providers recognize major contributions to CMM by CPS, and CPS are largely welcomed by primary care teams. These findings echo those of other reports of CPS integrating into primary care teams. ${ }^{41-46}$ However, interviews with CPS and team members demonstrate that the degree and quality of CPS' integration varied across teams in nuanced ways. We found that some teams adopted the CPS in more of an auxiliary role, which we characterize as consultative; other teams adopted the CPS in a more fully developed role, which we characterize as collaborative integration. When CPS work in a fully collaborative role, they work to the top of their scope of practice, which in the VHA means performing CMM when seeing patients independently between PCP visits and managing a range of diseases collaboratively with a PCP. In this role, they can improve access, lower costs, and reduce PCP burden. ${ }^{19,47} \mathrm{By}$ contrast, consultative integration is partial integration. While a CPS who is seen as auxiliary support for PCPs might free up PCP time by answering questions rapidly, ${ }^{48}$ positioning CPS as medication consultants uses only part of their skillset.

Team members' attitudes about PCP patient ownership may be a barrier to fully collaborative integration. We see evidence of such attitudes in the quotes above, where a PCP describes a concern about having "too many cooks in the kitchen." In the early years of the VHA's reorganization of primary care into PACT, team members expressed mixed feelings about CPS joining teams, citing reservations about CPS' knowledge and abilities. ${ }^{49}$ Even 2 years after the implementation of CPS on interprofessional teams, PCPs continued to feel solely responsible for most tasks, ${ }^{15}$ suggesting that an ethos of physician ownership over patients can be a barrier to achieving interprofessional collaboration. ${ }^{50}$ Our findings also echo some Australian pharmacists' perceptions that PCPs deny their skills and dismiss their advice, which researchers interpreted as a demarcation of medical territory and an expression of "medical dominance." ${ }^{51}$ On the other hand, PCPs have the opportunity to use their positional power to establish the CPS' collaborative role. ${ }^{9,10}$

Team members' misconceptions about the CPS' scope of practice and training, or lack of definition of the CPS' role, may also be a barrier to CPS' collaborative integration. In the VHA, although 51\% of VHA pharmacists had a scope of practice authorizing them to work as advanced practitioners in $2019,{ }^{18}$ pharmacists also historically and presently perform medication distribution and lead diseaseor medication-specific clinics, and some CPS split their time between roles as team members and direct providers. ${ }^{52}$ CPS specializing in primary care CMM may be misperceived as having either the well-known traditional role of medication distribution or the role of heading a disease-specific clinic (eg, anticoagulation clinics). Insufficiently defined roles have been cited by PCPs, in Australia, as a reason for their mixed views on the prospect of CPS joining their teams, while those PCPs who had prior experience working with CPS in the role of an advanced practice provider expressed fewer reservations. ${ }^{43}$ A realist review of the literature also found that PCPs' acceptance of clinical pharmacists increased after they had experience working with a pharmacist in general practice, and likewise, pharmacists need time to demonstrate their value. ${ }^{53}$ For example, Canadian clinics that added primary care pharmacists provide some evidence of newly-introduced CPS fluctuating between feeling underutilized and highly valued, ${ }^{54}$ and teams finding it helpful to assess, after the initial integration, alignment between the desired and established role. ${ }^{55}$

\section{Limitations}

The CPS in this study work in multiple teamlets within PACT at each medical center, ${ }^{2}$ which means that 1 CPS may experience multiple types of integration. For this reason, we did not conduct a sitelevel analysis and our findings cannot address how some facility-specific factors impacted CPS' integration (eg, the geographic location of the clinics [west coast; Midwest; east coast], their degree of rurality; the unique aspects of the medical centers' implementation of the nationwide initiative; individual PCPs' experience with CPS). Conclusions 
we draw about CPS' integration may not be generalizable to the experiences of other teams or health care systems, although we anticipate that many of the concepts and findings discussed here may be transferable to other studies on interprofessional teamwork and team integration. ${ }^{56}$ Surveying a large sample of CPS and team members would reveal more about the prevalence of these different perceptions about CPS' role in primary care, and such a survey is part of our larger evaluation. ${ }^{3-35}$ This study is a snapshot of teams' early experiences with clinically trained pharmacists. There is some evidence that CPS' role becomes more defined over time,${ }^{54,55}$ and we expect it is possible for CPS who were integrated into consultative roles to shift into collaborative roles that allow them to practice to the top of their scope. The relationship between consultative and collaborative integration is unknown, and so are the mechanisms by which the CPS' role eventually becomes agreed on, and these are areas for further investigation. As our ongoing evaluation of the CRVA initiative continues, we will be able to assess how the CPS role evolves. Finally, this study does not explore the relationship between CPS' integration and patient health outcomes, or patients' perceptions of care. These are important areas for further research.

\section{Conclusion}

Nationally ${ }^{41,42,55}$ and internationally, ${ }^{51,53,54,57,58}$ there is a movement to diversify CPS' duties and incorporate them into PCMH. CPS can be an asset for primary care practices, but to maximize their contributions as direct care providers, a key part of team expansion is clarifying the clinically trained pharmacist's role. This study describes the process of incorporating a primary care pharmacist into VHA PACT. We conclude that efforts to add CPS to primary care teams should anticipate barriers to achieving fully collaborative integration, which may include team members' attitudes about PCP patient ownership and misconceptions about the CPS' optimized role. Team expansion needs to be preceded or accompanied by some educational outreach. The expansion of team members' perceptions of the joining profession must be co-occurring with team expansion. These results can be used to inform efforts to expand clinical teams through deliberate, well-thought-out approaches to introduce new advanced practice providers, like CPS.
The authors gratefully acknowledge the primary care providers, pharmacists, and clinical team members who participated in this evaluation. This work was supported by the US Department of Veterans Affairs Office of Rural Health and the Clinical Pharmacy Practice Office of the Pharmacy Benefits Management Service. The views expressed in this article are those of the authors and do not necessarily represent the position or policy of the Department of Veterans Affairs or the US government.

To see this article online, please go to: bttp://jabfm.org/content/ 34/2/320.full.

\section{References}

1. American Academy of Family Physicians, American Academy of Pediatrics, American College of Physicians, American Osteopathic Association. Joint Principles of the Patient-Centered Medical Home 2007. Available at: https://www.aafp.org/dam/AAFP/documents/practice management/pcmh/initiatives/PCMHJoint.pdf. Accessed October 7, 2020.

2. Rodriguez HP, Giannitrapani KF, Stockdale S, Hamilton AB, Yano EM, Rubenstein LV. Teamlet structure and early experiences of medical home implementation for veterans. J Gen Intern Med 2014;29 Suppl 2:S623-31. Jul.

3. Rosland AM, Nelson K, Sun H, et al. The patientcentered medical home in the Veterans Health Administration. Am J Manag Care 2013;19:e26372. Jul 1.

4. Piette JD, Holtz B, Beard AJ, Ann Arbor PACT Steering Committee, et al. Improving chronic illness care for veterans within the framework of the PatientCentered Medical Home: experiences from the Ann Arbor Patient-Aligned Care Team Laboratory. Transl Behav Med 2011;1:615-23. Dec.

5. Landon BE, Gill JM, Antonelli RC, Rich EC. Prospects for rebuilding primary care using the patient-centered medical home. Health Aff (Millwood) May 2010;29:827-34.

6. Karam M, Brault I, Van Durme T, Macq J. Comparing interprofessional and interorganizational collaboration in healthcare: a systematic review of the qualitative research. Int J Nurs Stud 2018;79:70-83.

7. Xyrichis A, Lowton K. What fosters or prevents interprofessional teamworking in primary and community care? A literature review. Int J Nurs Stud 2008;45:140-53. Jan.

8. Brault I, Kilpatrick K, D'Amour D, et al. Role clarification processes for better integration of nurse practitioners into primary healthcare teams: a multiple-case study. Nurs Res Pract 2014;170514 (Article ID).

9. MacNaughton K, Chreim S, Bourgeault IL. Role construction and boundaries in interprofessional primary health care teams: a qualitative study. BMC Health Serv Res 2013;13:486.

10. Schot E, Tummers L, Noordegraaf $M$. Working on working together. A systematic review on how 
healthcare professionals contribute to interprofessional collaboration. J Interprof Care 2020;34:33242. Jun.

11. Harrod M, Weston LE, Robinson C, Tremblay A, Greenstone CL, Forman J. "It goes beyond good camaraderie": a qualitative study of the process of becoming an interprofessional healthcare "teamlet". J Interprof Care May 2016;30:295-300.

12. Giannitrapani KF, Soban L, Hamilton AB, et al. Role expansion on interprofessional primary care teams: Barriers of role self-efficacy among clinical associates. Healthc (Amst). Dec 2016;4:321-6.

13. True G, Stewart GL, Lampman M, Pelak M, Solimeo SL. Teamwork and delegation in medical homes: primary care staff perspectives in the Veterans Health Administration. J Gen Intern Med 2014;29 Suppl 2:S632-9. Jul.

14. Solimeo SL, Ono SS, Lampman MA, Paez MB, Stewart GL. The empowerment paradox as a central challenge to patient centered medical home implementation in the Veteran's Health Administration. J Interprof Care 2015;29:26-33. Jan.

15. Edwards ST, Helfrich CD, Grembowski D, et al. Task delegation and burnout trade-offs among primary care providers and nurses in Veterans Affairs Patient Aligned Care Teams (VA PACTs). J Am Board Fam Med 2018;31:83-93.

16. Edwards ST, Rubenstein LV, Meredith LS, et al. Who is responsible for what tasks within primary care: perceived task allocation among primary care providers and interdisciplinary team members. Healthc (Amst). Sep 2015;3:142-9.

17. Tuepker A, Kansagara D, Skaperdas E, et al. "We've not gotten even close to what we want to do": a qualitative study of early patient-centered medical home implementation. J Gen Intern Med 2014;29 Suppl 2:S614-22.

18. McFarland MS, Groppi J, Jorgenson T, et al. Role of the US Veterans Health Administration clinical pharmacy specialist provider: shaping the future of comprehensive medication management. Can J Hosp Pharm 2020;73:152-8.

19. McFarland MS, Nelson J, Ourth H, Groppi J, Morreale A. Optimizing the primary care clinical pharmacy specialist: increasing patient access and quality of care within the Veterans Health Administration. J Am Coll Clin Pharm 2020;3:494-500.

20. Ourth H, Groppi J, Morreale AP, Quicci-Roberts $\mathrm{K}$. Clinical pharmacist prescribing activities in the Veterans Health Administration. Am J Health Syst Pharm 2016;73:1406-15.

21. Groppi JA, Ourth H, Morreale AP, Hirsh JM, Wright S. Advancement of clinical pharmacy practice through intervention capture. Am J Health Syst Pharm 2018;75:886-92.

22. Seckel E, Portillo E, Lehmann M, Wilcox A, Vega R. Ambulatory care pharmacy practice advan- cement: diffusing strong practices. The Journal of the Pharmacy Society of Wisconsin. July August 2019;22:58-61.

23. The Patient-Centered Primary Care Collaborative. The Patient-Centered Medical Home: Integrating Comprehensive Medication Management to Optimize Patient Outcomes. 2012. Available at: https://www. pcpcc.org/sites/default/files/media/medmanagement. pdf. Accessed June 24, 2020.

24. Talon B, Perez A, Yan C, et al. Economic evaluations of clinical pharmacy services in the United States: 2011-2017. Journal of the American College of Clinical Pharmacy 2020;3:793-806.

25. Edwards HD, Webb RD, Scheid DC, Britton ML, Armor BL. A pharmacist visit improves diabetes standards in a patient-centered medical home (PCMH). Am J Med Qual 2012;27:529-34.

26. Jameson JP, Baty PJ. Pharmacist collaborative management of poorly controlled diabetes mellitus: a randomized controlled trial. Am J Manag Care 2010;16:250-5.

27. Cioffi ST, Caron MF, Kalus JS, Hill P, Buckley TE. Glycosylated hemoglobin, cardiovascular, and renal outcomes in a pharmacist-managed clinic. Ann Pharmacother May 2004;38:771-5.

28. Hirsch JD, Steers N, Adler DS, et al. Primary care-based, pharmacist-physician collaborative medication-therapy management of hypertension: a randomized, pragmatic trial. Clin Ther 2014;36:1244-54. Sep 1.

29. Lee JK, Grace KA, Taylor AJ. Effect of a pharmacy care program on medication adherence and persistence, blood pressure, and low-density lipoprotein cholesterol: a randomized controlled trial. JAMA 2006;296:2563-71.

30. Galewitz P. Clinical pharmacists employed by VA have increased nearly $50 \%$ in 5 years. Pharmacy Today 2017;23: p 6-7.

31. McFarland MS, Lamb K, Hughes J, Thomas A, Gatwood J, Hathaway J. Perceptions of Integration of the Clinical Pharmacist into the Patient Care Medical Home Model. J Healthc Qual 2018;40: 265-73.

32. Farrell B, Pottie K, Woodend K, et al. Developing a tool to measure contributions to medicationrelated processes in family practice. J Interprof Care 2008;22:17-29.

33. McCullough MB, Gillespie C, Kleinberg F, et al. Challenges of clinical pharmacy specialists' role expansion to improve access to PACT and pain management: A mixed methods formative evaluation. Poster presented at: US Department of Veterans Affairs Health Services Research \& Development/QUERI National Meeting October 29-31 2019; Washington, DC.

34. McCullough MB, Lee $\mathrm{K}$, Kleinberg $\mathrm{F}$, et al. Evaluating the integration of clinical pharmacy 
specialists in primary care to improve access to care for rural Veterans. Poster presented at: American Pharamcists Association Annual Meeting; March 22-25 2019; Seattle, WA.

35. McCullough MB, Lee $\mathrm{K}$, Kleinberg F, et al. The introduction of clinical pharmacy specialists in primary care: Evaluating the integration of a new clinical team member in a complex intervention. Poster presented at: 11th Annual Conference on the Science of Dissemination and Implementation in Health December 3-5 2018; Washington, DC.

36. Guest G, Bunce A, Johnson L. How many interviews are enough?: An experiment with data saturation and variability. Field Methods 2006;18: 59-82.

37. Boyatzis RE. Transforming Qualitative Information: Thematic Analysis and Code Development. SAGE 1998.

38. Miles MB, Huberman AM. Qualitative Data Analysis: An Expanded Sourcebook 2nd ed. SAGE Publications, Inc.; 1994.

39. Ryan GW, Bernard HR. Techniques to identify themes. Field Methods 2003;15:85-109.

40. Clarke V, Braun V, Hayfield N. Thematic Analysis. In: Smith JA, ed. Qualitative Psychology: A practical guide to research methods $3 \mathrm{rd}$ ed. SAGE Publications Inc.; 2015:222-48.

41. Smith M, Bates DW, Bodenheimer T, Cleary PD. Why pharmacists belong in the medical home. Health Aff (Millwood) May 2010;29:906-13.

42. Choe HM, Farris KB, Stevenson JG, et al. Patientcentered medical home: developing, expanding, and sustaining a role for pharmacists. Am J Health Syst Pharm 2012;69:1063-71. Jun 15.

43. Nigro SC, Garwood CL, Berlie H, et al. Clinical pharmacists as key members of the patient-centered medical home: an opinion statement of the Ambulatory Care Practice and Research Network of the American College of Clinical Pharmacy. Pharmacotherapy 2014;34:96-108. Jan.

44. Chisholm-Burns MA, Kim Lee J, Spivey CA, et al. US pharmacists' effect as team members on patient care: systematic review and meta-analyses. Med Care 2010;48:923-33. Oct.

45. Bunting BA, Smith BH, Sutherland SE. The Asheville Project: clinical and economic outcomes of a community-based long-term medication therapy management program for hypertension and dyslipidemia. J Am Pharm Assoc 2008;48:23-31. Jan-Feb.
46. Moreno G, Lonowski S, Fu J, et al. Physician experiences with clinical pharmacists in primary care teams. J Am Pharm Assoc (2003) 2017;57:686-91.

47. Rose AJ, McCullough MB, Carter BL, Rudin RS. The clinical pharmacy specialist: part of the solution. J Gen Intern Med 2017;32:375-7. Apr.

48. Funk KA, Pestka DL, Roth McClurg MT, Carroll JK, Sorensen TD. Primary care providers believe that comprehensive medication management improves their work-life. J Am Board Fam Med 2019;32:462-73.

49. Patterson BJ, Solimeo SL, Stewart KR, Rosenthal GE, Kaboli PJ, Lund BC. Perceptions of pharmacists' integration into patient-centered medical home teams. Res Social Adm Pharm 2015;11:85-95.

50. Zink T, Kralewski J, Dowd B. The transition of primary care group practices to next generation models: satisfaction of staff, clinicians, and patients. J Am Board Fam Med. 2017;30:16-24.

51. Luetsch K, Scuderi C. Experiences of medical dominance in pharmacist-doctor interactions - An elephant in the room? Res Social Adm Pharm Dec 16 2019.

52. McCullough MB, Solomon JL, Petrakis BA, et al. Balancing collaborative and independent practice roles in clinical pharmacy: a qualitative research study. Ann Pharmacother 2015;49:189-95. Feb.

53. Anderson C, Zhan K, Boyd M, Mann C. The role of pharmacists in general practice: A realist review. Res Social Adm Pharm 2019;15:338-45.

54. Dolovich L, Pottie K, Kaczorowski J, et al. Integrating family medicine and pharmacy to advance primary care therapeutics. Clin Pharmacol Ther 2008;83:913-7.

55. Kolodziejak L, Remillard A, Neubauer S. Integration of a primary healthcare pharmacist. J Interprof Care May 2010;24:274-84.

56. Curry LA, Krumholz HM, O'Cathain A, Plano Clark VL, Cherlin E, Bradley EH. Mixed methods in biomedical and health services research. Circ Cardiovasc Qual Outcomes 2013;6:119-23. Jan 1.

57. Tan EC, Stewart K, Elliott RA, George J. Integration of pharmacists into general practice clinics in Australia: the views of general practitioners and pharmacists. Int $\mathrm{J}$ Pharm Pract 2014;22:28-37.

58. Tan EC, Stewart K, Elliott RA, George J. Pharmacist services provided in general practice clinics: a systematic review and meta-analysis. Res Social Adm Pharm 2014;10:608-22. 